Fragmento de Las partículas contra los torrentes.

Técnica óleo. $130 \times 195$ cm. 2003 Diego Mazuera

\title{
EL ESTADO DE LA INFRAESTRUCTURA EN COLOMBIA FRENTE AL RETO DE LA GLOBALIZACIONN
}

Dr. Luis Fernando Jaramillo Correa

\section{RESUMEN}

El principal obstáculo de Colombia para su desarrollo económico ha sido la deficiente infraestructura y muy especialmente su red de carreteras. El disponer de centros de producción lejanos a los puertos ocasiona un costo alto para el transporte de mercancías de exportación y de materias primas de importación. Adicionalmente, más del $60 \%$ de sus carreteras se encuentran en mal estado. Esto genera problemas serios para competir exitosamente en los mercados externos, ya que el costo y la calidad de los servicios de transporte afectan la competitividad, el desarrollo, la integración económica y las posibilidades de inserción en el mundo globalizado.

Un componente importante de la estrategia exportadora del país está relacionado con la necesidad de poseer una infraestructura adecuada y eficiente, que permita generar mayores niveles de productividad vía menores costos de transporte y servicios logísticos.

Luego de los 10 años de apertura económica que han transcurrido, se ha incrementado en forma sustancial la demanda de infraestructura regional. Empero, sólo se dedica una inversión del orden del 0.9 por ciento del PIB a la construcción y mantenimiento de carreteras, monto que se encuentra muy por debajo del promedio de la región, que se sitúa en el 2.0. Con urgencia se requiere de manera continua aumentar la inversión en carreteras del orden del 4.0 por ciento del PIB.

A continuación se hace una síntesis del estado actual de la infraestructura en Colombia y se analiza el plan de desarrollo del actual gobierno y las prioridades del Ministro del Transporte en materia de infraestructura. Finalmente, se brindan recomendaciones para incrementar la disponibilidad de obras de infraestructura que le permita a Colombia enfrentar exitosamente el camino de la globalización.

\section{ABSTRACT}

The main obstacle to Colombia for its economical development has been the deficient infrastructure and especially its net of roads. Having production centers far from ports causes a high cost for transportation of export merchandise and raw materials for import. In addition, more than 60\% of its roads are in bad condition. This brings about severe problems to compete successfully in the external markets since cost and quality of transportation services affect the competitiveness, development, economical integration and the possibilities of insertion in the globalized world.

An important component of the exporting strategy of the country related to the necessity of having and efficient and suitable infrastructure that allows generating greater levels of productivity via less transportation costs and logistic services.

After passing 10 years of the economical opening, the demand of regional infrastructure has increased substantially. However, just an investment of the order of 0.9 percent of IGP (internal gross product) is devoted to building and road maintenance, amount that is under the average of the region, which is located in the 2.0 . It is required urgently a continuous way to augment the investment on roads of the order of 4.0 percent of the IGP.

Following there is a synthesis of the current condition of Colombia's infrastructure and an analysis of the development plan of the present government and the priorities of the Transport Minister with regard to infrastructure. Finally, recommendations are offered to increase the availability of infrastructure works that allow Colombia to successfully deal with the way of globalization. 
En el actual marco de apertura económicay globalización, se hace necesario replantear el sistema de transporte en el país, para lograr niveles de costos que hagan posible la competencia de los productos colombianos en el escenario de los mercados internacionales

\section{INTRODUCCIÓN}

Uno de los principales obstáculos que ha tenido Colombia en su desarrollo económico, desde que surgió como Estado soberano, ha sido su deficiente infraestructura y muy especialmente su red de carreteras. El hecho de disponer de los centros de producción alejados de los puertos conlleva, de por sí, un costo muy alto para el transporte de mercancías de exportación y de materias primas de importación, que aunado al hecho de que algo más del $60 \%$ de sus carreteras se encuentran en mal estado de mantenimiento, la lleva a enfrentar serios problemas para competir con éxito en los mercados externos. El costo y la calidad de los servicios de transporte tienen una creciente relevancia para la competitividad, el desarrollo, la integración económica y la inserción dentro de un mundo globalizado.

Es por tanto oportuno recordar, que gran parte de la estrategia exportadora está relacionada con la necesidad de poseer una infraestructura adecuada y eficiente, que permita generar mayores niveles de productividad vía menores costos de transporte y servicios logísticos, los cuales generen mejores niveles de competitividad y crecimiento. En el actual marco de apertura económica y globalización, se hace necesario replantear el sistema de transporte en el país, para lograr niveles de costos que hagan posible la competencia de los productos colombianos en el escenario de los mercados internacionales. La infraestructura reviste pues una enorme importancia para garantizar el desarrollo económico de Colombia.

Después de diez años de apertura económica, se ha incrementado en forma sustancial la demanda de infraestructura regional, lo cual exige un mejoramiento contundente en la forma como los sectores público y privado están atendiendo este reto. No obstante, la respuesta colombiana es muy tímida, al dedicar del orden del 0.9 por ciento del PIB a la construcción y mantenimiento de carreteras, monto que se encuentra muy por debajo del promedio de la región la cual sitúa en el 2.0 por ciento del PIB. Esto significa que en promedio, Colombia está realizando menos inversión de la que se considera adecuada en el resto de continente con la consecuencia de que cada día nos encontramos más rezagados en la estructuración de una red de transporte eficiente y competitiva.

El estado actual de la infraestructura colombiana es deficiente y colocarla en los niveles que requiere para hacer frente al reto de la globalización, a los nuevos órdenes hemisféricos y escenarios mundiales, requiere un aumento de la inversión continua en carreteras del orden del 4.0 por ciento del PIB, por lo menos durante los próximos cinco años, aspecto que desafortunadamente no se encuentra claro en el nuevo plan de desarrollo ni se ve posible dadas las restricciones macroeconómicas del país.

A continuación se hace una síntesis del estado actual de la infraestructura en Colombia. Posteriormente, se analiza el plan de desarrollo y al final, se brindan recomendaciones para incrementar la disponibilidad de obras de infraestructura que le permitan a Colombia enfrentar exitosamente el camino de la globalización.

\section{LA INFRAESTRUCTURA VIAL}

Cuando se examina el estado actual de la infraestructura colombiana y su capaci- 
dad de responder frente al reto de la globalización, se hace evidente que Colombia es un país que tradicionalmente ha tenido enormes problemas en su infraestructura. La misma topografía constituye un reto a la integración, ya que es un país de regiones, no por vocación etnográfica o antropológica, sino por la dificultad intrínseca de comunicar a las diversas regiones.

A mediados del siglo pasado comenzó el proceso de integración vial, cuando se construyen carreteras troncales, que son las primeras vías de comunicación importantes en muchos años distintas de los ferrocarriles y del río Magdalena. Sin embargo, en ese proceso de mutación del transporte ferroviario y fluvial en transporte carretero, se produce la decadencia total de los ferrocarriles y la reducción a un mínimo del transporte fluvial, mientras se daba la concentración en el transporte carretero del movimiento de carga y la mayor parte del movimiento de pasajeros.

Este proceso vial avanza a través de los años configurando una red troncal de carreteras, de nivel intermedio, la cual no constituye un sistema eficiente que esté vinculando a los grandes centros de pro-

\section{RESEÑA DE AUTOR}

Ingeniero civil de la Universidad Nacional de Colombia, con estudios en London School of Economics. Ha desempeñado diversos cargos en la empresa privada como la ANDI, el INCORA, AVIANCA, Grancolombiana de Ingeniería y Construcciones. GRANDICÓN S.A., y el BID, Y cargos públicos como Ministro de Obras Públicas y Transporte, Ministro de Relaciones Exteriores, Canciller, Embajador ante la Organización de las Naciones Unidas, y el Reino de España.

Presidente de la Dirección Nacional del Partido Liberal Colombiano, Presidente del Consejo de Representantes del Centro Sur.

Actualmente es Miembro del Consejo Asesor Empresarial del Ministerio de Comercio Industria y Turismo para la negociación del TLC con los Estados Unidos de América. ducción y a las grandes ciudades ${ }^{1}$ con los puertos marítimos vitales para la comunicación con el exterior.

En años más recientes, durante la administración del Presidente Samper, se inicia vigorosamente el sistema de concesiones viales; una inversión cuantiosa para ejecutar obras con ayuda del sistema financiero y con aportes de capital de riego de los concesionarios. Esto permitió que durante dicho período se invirtieran en el país más de tres billones de pesos en mejoramiento de las vías troncales carreteras que unen los centros de producción con los principales puertos y se sentaran las bases para la reconstrucción de la red ferroviaria principal.

Sin embargo, después de ese importante esfuerzo de mitad de la década anterior, durante la presidencia de Andrés Pastrana se avanza muy poco; la contratación pública incuba problemas y dificultades que nunca se resuelven en el tiempo y la solución propuesta por parte de los funcionarios públicos es la "judicialización" de todos los problemas, produciendo un efecto nocivo para la credibilidad del sistema de concesiones y un mal sabor en la opinión pública al producirse numerosos fallos de los tribunales de arbitramiento en contra de los intereses del gobierno. Se produce desdichadamente un retroceso en todo el proceso de modernización de la red vial que es muy conveniente anotar.

La experiencia muestra que los temas de controversia se discuten por años sin llegar a solución alguna, hasta que finalmente se tiene que acudir a los controvertidos tribunales de arbitramento para evitar una paralización total de los sistemas y la denegación

1 Como sería el triángulo de oro Bogotá, Cali, Medellín. 
Hoy resulta más costoso llevar una tonelada por carretera de Bogotá a un puerto, que del puerto a cualquier lugar del mundo. de justicia. Luego viene la crítica por las decisiones de los tribunales, cuyo fallo en derecho no puede ser distinto dado las causas que son sometidas a su consideración.

Colombia no está realmente preparada para poder competir, máxime cuando una de las características de nuestro país es que los principales centros de producción y de generación de riqueza están en los Andes. Bogotá produce más del $50 \%$ del producto interno bruto nacional y es el principal centro industrial y comercial en servicios del país. Y las tres ciudades principales Bogotá, Cali, Medellín, que representan más del 80\% del PIB colombiano, tienen vías de comunicación precarias con los puertos. Y estas hay que hacerlas eficientes sobre cualquier otra prioridad o consideración en el sector, de lo contrario, estaríamos afectando seriamente la capacidad de competencia y de competitividad del país.

La carretera de Bogotá a Buenaventura (primer puerto de país y el principal para Bogotá) debería ser una autopista de primer orden. Con la vía hacia la Costa Atlántica acontece algo similar. En el último gobierno se frustró la construcción de la nueva vía entre Bogotá y Puerto Salgar que produciría importantes ahorros en la comunicación con Medellín y con la costa por un nuevo trazado. Hoy resulta más costoso llevar una tonelada por carretera de Bogotá a un puerto, que del puerto a cualquier lugar del mundo. Mientras en la carretera Bogotá - Villavicencio que comunica a la capital con los Llanos Orientales -sin dejar de valorar lo importante que es el llano- se han invertido cerca de un billón de pesos. Todo esto nos deja serias dudas sobre la forma en la cual se fijan las prioridades del país.

Colombia necesita hacer un esfuerzo importante en la conexión de Bogotá con Buenaventura, que, como lo hemos dicho, es su principal puerto y en la actualidad existen estudios y varias alternativas para construir una vía de primer orden con características de autopista. Por lo tanto, debemos abocarnos al estudio de una opción definitiva que incluya la construcción de un túnel en La Línea definido con seriedad y pensando en los mejores intereses del país. El ahorro que obtiene el país construyendo una obra de la magnitud del túnel de La Línea es significativo, pero si se va a construir en lo alto de la cordillera a 2.600 metros, cualquier inversión allí sería desaprovechada ${ }^{2}$. Hay que abocar nuevamente el estudio con toda seriedad, para poder ratificarle al país que Bogotá hay que conectarla eficientemente con los dos océanos mediante vías de comunicación que bajen sustancialmente los costos del transporte. El resto de ciudades capitales principales también hay que unirlas en forma prioritaria y urgente con vías de especificaciones de primer nivel.

Otro aspecto fundamental, -aparte de las diferencias que tengamos con Venezuela y su gobierno actual y sus amores y desamores con Colombia-, es que necesariamente vamos a desarrollar un mercado importante con Venezuela, por la complementación que se presenta necesariamente entre nuestras economías y para eso se necesita construir la auto-

2 Salir de Bogotá a 2.600 metros de altura, bajar a Girardot a orillas del rió Magdalena a 400 metros, subir otra vez al túnel propuesto para La Línea a 2.600 metros, para volver a bajar al nivel del mar, es un despropósito. 
pista de los Llanos ${ }^{3}$. Esta carretera ahorraría cerca de la mitad del tiempo que utiliza una tractomula entre Caracas y Bogotá, que en la actualidad es de 36 horas. El tiempo de viaje se reduciría a unas 18 horas aproximadamente, disminuyendo sensiblemente los costos de transporte. Esta es otra de las obras críticas que tiene que hacer Colombia.

Otro proyecto indispensable es la vía de comunicación con Quito - no a través del Valle del Cauca, Cauca y Nariño que representa altos costos de transporte - sino mejorar el trazado Bogotá - Neiva - Pitalito - Mocoa - Puente San Miguel una obra que está avanzada, pero que hay que terminarla como una vía troncal que nos comunique con el Ecuador, otro de nuestros mercados importantes.

El mercado americano y el mercado europeo seguirán siendo los más importantes del país y debemos tener las vías de comunicación y de exportación con los puertos operando en una forma adecuada. Pero es indudable que hacia el futuro, la relación con Ecuador y con Venezuela va a ser una parte significativa de nuestro comercio exterior pero hay que estimularlo, abaratarlo y hacerlo eficiente para que tenga mayor beneficio para las partes.

Otro problema grave que tiene el sector de la infraestructura vial de carreteras viene desde la desaparición del Fondo Vial Nacional con la Constitución del 91, cuando se crea la unidad de caja de los dineros del gobierno bajo la inspiración del Ministro de Hacienda de esa época. Con el principio de unidad de caja se sacrifica una serie de elementos importantes en el desarrollo colombiano, al punto que Colombia hoy se encuentra por debajo del $0.7 \%$ del PIB en inversión en infraestructura, cuando prácticamente el resto de los países importantes de América Latina están por el 2\% de inversión anual del PIB en infraestructura. Los recursos dedicados al mantenimiento de la red en los últimos diez años están bien por debajo de los niveles que se podrían considerar como los mínimos necesarios.

Es decir, se tiene un rezago importante y creciente. Cada vez invertimos menos, cada vez tenemos mayor dispersión en los propósitos y en las metas de inversión en infraestructura. En consecuencia el rezago va a ser progresivo y esto es aún más grave cuando una de las primeras manifestaciones del gobierno nacional es que los concesionarios son contratistas dedicados a defraudar al Estado. Después de una declaración de ese tenor va a ser difícil movilizar recursos del sector financiero hacía un sistema concesionado integrado por gentes "inescrupulosas". Luego, hay que entrar en lento y penoso proceso de reconstrucción de credibilidad para que el Estado pueda contar con el sector privado y muy especialmente el sector financiero como fuente de recursos en vista de las dificultades fiscales crónicas que aquejan al gobierno colombiano. Al fin y al cabo el concecionario es simplemente un intermediario entre el sector financiero y el estado.

Colombia estaba más y mejor preparada para exportar, desde el punto de vista de la red de carreteras, hace cuatro lustros de lo que se encuentra ahora. Esto se debe en especial a que aún cuando se realizaron inversiones importantes en carreteras durante las
Colombia estaba más y mejor preparada para exportar, desde el punto de vista de la red de carreteras, hace cuatro lustros de lo que se encuentra ahora.

3 A la fecha está construida una carretera pavimentada entre Bogotá - Villavicencio - Yopal - Hato Corozal y faltan 150 kilómetros aproximadamente para estar conectados con Arauca. Venezuela está trabajando entre Guadualito, Barinas y Caracas y con eso se tendría una vía prácticamente plana, trazada por el pie de monte de la cordillera. 
décadas de los 60 y 70, como se mencionó se invirtieron pocos recursos en su mantenimiento. ${ }^{4}$ En consecuencia, en nuestro país se ha perdido el concepto de "patrimonio vial". Esta descarriada patrimonial, ha hecho que los recursos estatales para la construcción de nuevas vías se hicieran escasos y el mantenimiento no fuera adecuado, por lo cual se creó un déficit vial que hizo indispensable la participación privada en la construcción, mantenimiento y operación de las carreteras, mediante el sistema de concesiones.

En época del Presidente Virgilio Barco, el país gozaba de una malla vial secundaria y terciaria aceptable, pero la posterior transferencia a los entes territoriales sin tecnología y recursos llevó al deterioro sustancial de las vías de penetración, haciendo onerosa la salida de productos agropecuarios. Además, el resultado de los años de abandono es tan alarmante que cerca del $45 \%$ de la red de carreteras no puede prestar el servicio previsto ${ }^{5}$. Es conveniente que el país avance también en la mejoría de su infraestructura vial secundaria y terciaria con un programa subsidiario que se tiene que hacer necesariamente, pero no puede ser el programa bandera de un ministerio. El programa tiene que estar enfocado a resolver los problemas económicos del país, a colocar a Colombia como ente com- petitivo, como jugador dentro del contexto internacional.

La necesidad de modernizar y ampliar la red de carreteras es un imperativo para la competitividad colombiana en los nuevos escenarios del libre comercio. A pesar de que temas como el G3, la CAN, el ALCA, el TLC, la OMC, se vienen mencionando desde hace más de cinco años, Colombia no se ha anticipado al cambio, ni tiene proyecciones al futuro en materia de infraestructura física frente al reto de la globalización ${ }^{6}$.

Por tanto, se requiere retomar la confianza en el sistema de concesiones ante la falta de recursos fiscales. Se requiere brindar mecanismos y garantías que aseguren el uso de buena parte de los fondos de pensiones, revaluando las matrices de riesgos y la asignación de los mismos. Así como honrar los compromisos con las concesiones actuales para que el sector privado, tanto el inversionista como el financiero, recupere la confianza y vuelva a invertir en el sistema vial.

Por lo demás, se deben complementar los flujos privados con movilización de recursos públicos, en inversión física, y preinversión (estudios, diseños, ingeniería, etc.), de manera que permita invertir los recursos estableciendo un orden de prioridades estricto ya que en el caso de las carreteras las

4 Las ventajas del mantenimiento son especialmente importantes en el sector vial, dado que una carretera se puede mantener por tiempo indefinido con un gasto anual de aproximadamente el $2 \%$ de su costo de construcción. Si se renuncia al mantenimiento, el costo de reconstrucción de la carretera implica un gasto superior a cinco veces el costo capitalizado de mantenimiento anual.

5 La infraestructura terrestre del país es de aproximadamente $114,000 \mathrm{~km}$. De éstos, el 57.6\% son vías veredales y municipales, el $22.4 \%$ nacionales y el $20 \%$ caminos vecinales. Comparada con los estándares internacionales, la infraestructura vial del país está muy poco desarrollada, pues sólo un $11 \%$ de las vías se encuentran pavimentadas.

6 El estado actual de la infraestructura vial de varios países latinoamericanos es deficiente en comparación con el resto del mundo. De acuerdo con el Banco Mundial, estos países tienen cerca de $1.000 \mathrm{~km}$. de vías pavimentadas por millón de habitantes (frente a $10.000 \mathrm{~km}$. por millón de habitantes en los países industrializados). En el caso colombiano no solo este número es mucho menor sino que una gran parte de ellas se encuentran en un precario estado de mantenimiento. 
cantidades invertidas en conservación son recuperadas y multiplicadas a través de la disminución de los costos de transporte ${ }^{7}$.

\section{LA INFRAESTRUCTURA FÉRREA}

Se puede afirmar que Colombia cuenta con el privilegio, de haber sido una de las primeras naciones del orbe que introdujo dentro de su territorio, el revolucionario sistema de transporte nacido del portentoso invento de la locomotora. En efecto, el propio Libertador, al estructurar su maravilloso sueño de unión de las Américas, le dio prelación a la comunicación interoceánica de la Gran Colombia de entonces, a través del istmo de Panamá, bien fuera a través de un canal oceánico o mediante la construcción de un "camino de rieles".

El deteriorado estado de la infraestructura ferroviaria a finales del siglo XX, indicaba que de $3.154 \mathrm{Km}$ que constituían la red ferroviaria del país, solo 1.915 kilómetros (61\%) estaban parcialmente activos y 1.239 kilómetros (39\%) estaban totalmente fuera de funcionamiento y eran irrecuperables.

Con respecto al sistema ferroviario hay un esquema de reconstrucción importante, puesto que hoy en día se están exportando alrededor de treinta millones de toneladas de carbón por ferrocarril ${ }^{8}$. Esto significa un cambio enorme del sistema ferroviario colombiano, que antes estaba destinado al transporte de pasajeros y de carga general. En la mejor de las épocas del ferrocarril se lograban transportar dos millones de toneladas anuales y hoy transportan treinta millones; la mira es llegar a sesenta millones de toneladas de carbón exportado por ferrocarril hacia el final de la década.

No obstante la perspectiva de un aumento significativo de la carga transportada, no se sustituyó la trocha férrea cuando la crisis fue total y no había carga que estuviera recorriendo las líneas férreas del país; en ese momento cuando se rehabilitaron totalmente los $220 \mathrm{Kms}$. del tramo carbonero se debió haber tomado la decisión de cambiar a trocha ancha de 1.45 metros, en vez de la trocha yárdica que se tiene hoy. Sin embargo, con la trocha de yarda el tren sigue siendo uno de los protagonistas importantes del transporte; hoy en día las locomotoras entre La Loma y Puerto Drummond están recorriendo la vía a una velocidad de 70 a 80 kilómetros por hora, mediante trenes de cien vagones con tres locomotoras.

Los ferrocarriles son un factor importante en el comercio de graneles, y en el manejo de carbón es prácticamente irremplazable. Todos los estudios que tenemos indican que la mejor opción para sacar los carbones en este momento es por medio del tren, pues el transporte que había, de la mina al sistema fluvial y de éste a los barcos de altamar, es más costoso de lo que es el ferrocarril en este momento. Hay necesidad entonces a muy corto plazo de empezar a programar la construcción de la doble línea entre los puertos de exportación y La Loma, de modo que provea una capacidad de transporte en ese corredor de cerca de cuarenta millones de toneladas por año. La línea actual permite exportar de forma óptima alrededor de

7 Se calcula que los sobrecostos operacionales de los vehículos y los costos de rehabilitación debidos al mal estado de los caminos representan gastos anuales innecesarios entre el 1.5\% y 3\% del PIB de los países latinoamericanos.

8 Quince millones en el Cerrejón más quince millones de toneladas de Drummond para finales del año 2003. 
Paragarantizar la interacción del modo férreo con el sistema de transporte nacionaly reducir los costos del mismo, la rehabilitación de la red se debe yuxtaponercon la habilitación de puertos secos y centros de transferencia intermodal de carga. veintidós millones de toneladas y va a estar copada muy rápidamente.

En el sistema ferroviario colombiano lo demás es secundario, puesto que el tren que va de la Dorada hasta Neiva difícilmente puede garantizar carga que justifique económicamente su recuperación o el ramal que comunica Puerto Berrío con Medellín solo ameritaría una rehabilitación ligera puesto en este sector la carga previsible es muy limitada. Pretender subir el tren a Bogotá es económicamente inviable ya que cada tren que llegue a La Dorada hay que subdividirlo en varios trenes y adicionar un número importante de locomotoras. Desde allí a Bogotá la única alternativa viable es el transporte multimodal utilizando el modo carretero y haciendo una transferencia de los contenedores en un puerto seco mecanizado y eficiente.

El paso siguiente en el proceso de modernizar el ferrocarril, y si se pretende conectar eficientemente el puerto con los centros principales, es no solo su rehabilitación, sino la modernización del sector de Cali a Buenaventura, mejorando el alineamiento de Dagua a Loboguerrero y construyendo alguna de las variantes que han sido estudiadas. Este sector del ferrocarril nunca va a ser muy rentable y de mucha eficiencia, pues la carga máxima que puede esperarse según los estimativos disponibles hoy es de millón y medio a dos millones de toneladas. Pensar en extender ese ferrocarril más allá no tiene justificación económica pues la carga que pudiera alimentar este sector no lo justificaría?
Actualmente se encuentran en operación por parte del sector privado dos concesiones ferroviarias, denominadas Concesión del Pacífico y Concesión del Atlántico. La primera, deberá reconstruir y operar $499 \mathrm{Km}$ y la segunda $1.484 \mathrm{Km}$, lo cual indica que el $63 \%$ de la red original del país se encuentra en concesión ${ }^{10}$. Así como el tren privado del Cerrejón, el único en Colombia que opera en trocha ancha.

No obstante el estado de recuperación actual por el sistema de concesiones que le augura al ferrocarril buenas posibilidades de enfrentar volúmenes de carga a costos competitivos, la red ferroviaria continúa inconexa y los sistemas de complementación intermodal son inexistentes, lo cual por el momento le resta competitividad a este modo de transporte para enfrentar el reto de la globalización. Hacia el futuro deberemos avanzar paralelamente en el proceso de integración con los distintos modos de transporte para garantizar su eficiencia. De modo complementario, para garantizar la interacción del modo férreo con el sistema de transporte nacional y reducir los costos del mismo, la rehabilitación de la red se debe yuxtaponer con la habilitación de puertos secos y centros de transferencia intermodal de carga. Esto permitirá justificar las enormes inversiones que se están realizando en la recuperación del modo ferroviario y le permitirá acceder a la carga de vocación ferroviaria que, debido a la ubicación de su origen o destino, no cuenta con acceso directo a la red férrea.

9 En este momento se exporta treinta millones de toneladas de carbón, mientras la totalidad del café que produce el país son seiscientas mil toneladas. Es decir, si se exportará todo el café por el ferrocarril no tendría un impacto significativo en el volumen de carga.

10 Se espera que el ferrocarril permita reducir hasta un 10\% del flete del transporte desde Bogotá a la costa y viceversa, con lo cual los productos colombianos se vuelven más competitivos en el exterior. 
Para efectos de mantener la continuidad de la red intermodal nacional, es importante que el Estado continúe rehabilitando y ejecutando aquellos otros tramos económicamente viables, financieramente sostenibles y técnicamente factibles, actividad que debe ser realizada directamente por el Estado. Aquí es importante indicar la necesidad de comunicar la red con el resto de puertos de la costa atlántica como son Barranquilla y Cartagena. De esta forma, la red férrea permitirá consolidar la conectividad comercial entre las regiones del centro del país y las costas, mejorando la competitividad de nuestros productos en el exterior.

Finalmente, en aras de mantener vivo el sueño bolivariano de integrar a las repúblicas andinas, se debe continuar con el estudio del trazado del ferrocarril que atraviese Venezuela, Colombia y Ecuador a lo largo del piedemonte llanero en la medida en que los cuidadosos estudios que se realicen prevean importantes flujos de carga de vocación ferroviaria que le den justificación económica a esta iniciativa.

El ferrocarril es un ente económico que tiene que generar utilidades o de lo contrario será muy difícil garantizar su operación y su continuidad a mediano y largo plazo. Tener el ferrocarril como elemento de colonización o de desarrollo sin carga y sin responder a una ecuación económica, generaría costos inadmisibles. Es agregarle una carga adicional a un Estado con las limitaciones fiscales que soporta el colombiano, como casi todos los estados de la región en este momento.

\section{ESTADO DE LA INFRAESTRUCTURA FLUVIAL Y MULTIMODAL}

Colombia no dispone de un sistema de navegación comercial interno ${ }^{11}$, ni multimodal de movilización de carga y de pasajeros, ni tampoco existe la reglamentación y la normatividad que brinde seguridad jurídica para que se constituyan y operen verdaderas empresas de transporte y logística multimodal. La interacción entre el modo portuario, férreo, carretero y fluvial no despega por falta de una política clara del gobierno central. Para ver nacer un sistema multimodal en el país, se hace necesario complementar el sistema con centros modernos de transferencia y despacho de carga, reduciendo los costos de operación en los puertos y expidiendo la reglamentación aduanera necesaria para garantizar la eficiencia del sistema integrado. ${ }^{12}$

A la fecha, el río Magdalena y el río Meta tienen estudios dispersos sobre tarifas y costos de los servicios, pero éstos no permiten cuantificar el verdadero impacto en la competitividad del país ${ }^{13}$. Los puertos fluviales que existen a lo largo del río están totalmente abandonados o en el mejor de los casos, algunos pocos, en proceso de recuperación, la infraestructura existente es escasa y cuando existe se encuentra deteriorada, la ausencia de señalización de brazos y canales navegables, a la vez que la carencia de indicación de las profundidades que de paso no han sido mantenidas, ha tenido como efecto que vaya perdiéndose la navegabilidad, incluso para plataformas de bajo calado.

11 La red fluvial en Colombia representa 18.000 kilómetros de ríos navegables.

12 Estudios de fletes entre los puertos de la costa y Bogotá indican que el sistema bimodal tren-camión es más competitivo que el sistema multimodal barcaza-tren-camión.

13 El tráfico total de carga por el río Magdalena alcanzó su punto máximo en 1976 con 3 millones ton/año y desde entonces ha disminuido, actualmente se transportan entre 2.0 y 2.5 millones de toneladas/año.

La interacción entre el modo portuario, férreo, carretero $y$ fluvial no despega por falta de una política clara del gobierno central. 
En el modo de transporte fluvial se tienen dudas sobre si puede ser realmente competitivo hacia el futuro. Hay que hacer un esfuerzo para restablecer la navegación en el río Magdalena en la parte baja ${ }^{14}$, ya que en la parte media y alta está cerrado prácticamente durante la mitad del año; los costos que tiene la incertidumbre de enviar barcazas con su remolcador por el río y quedar atascadas en un banco de arena o que no puedan pasar, son enormes y eso es lo que está sucediendo hoy. Existe el compromiso de un grupo de empresarios de comenzar a utilizar el río Magdalena, pero tienen problemas económicos, porque el río no funciona y su rehabilitación es un problema de mucho costo y de largo tiempo. De modo que, dada la deforestación de la cuenca y los problemas ecológicos, va ha ser muy difícil que vuelva a recuperar el perfil que tuvo en alguna oportunidad.

De forma complementaria se habla del transporte fluvial por el río Meta para finalmente salir al Orinoco. Allí hay posibilidades si Colombia tiene que seguir importando masivamente aceros de la Guyana Venezolana y traerlos hasta Puerto Gaitán, haciendo una adecuación del río Meta, que posee calado suficiente aunque limitado. Pero la navegación fluvial como se ve hoy, dentro de las características actuales del país, diría yo, que es una prioridad secundaria.

Finalmente, se deben consolidar empresas portuarias que busquen rescatar la navegabilidad del río Magdalena e incrementar la movilización de pasajeros, intensificar los volúmenes de carga transportada y rebajar los costos de los fletes.
Todos estos proyectos deben desarrollarse con la participación de la empresa privada como el camino más viable para conseguir el nivel de habilidad necesaria para afrontar los escenarios futuros, los cuales plantean una competencia abierta en mercados altamente competitivos.

\section{LA INFRAESTRUCTURA PORTUARIA}

En materia de infraestructura portuaria Colombia esta sobredimensionada. La carga impo/expo se podría mover hoy por Buenaventura y a través de puertos y en la Costa Atlántica. Las tarifas han disminuido a menos de la mitad, tal vez a un treinta y cinco por ciento y la operación portuaria, hoy en día, se realiza con un factor de eficiencia alto. El diagnóstico indica que no hay necesidad de nuevos puertos en el mediano plazo y en el caso de las propuestas que se han considerado en los últimos tiempos como el puerto de Tribugá y otras alternativas del Atlántico, la prueba de ácido que tienen que pasar es conseguir que alguien en el sector privado se comprometa con su construcción y operación mediante una concesión. Estoy seguro que no van a competir con puertos que están completamente amortizados y operando con algún grado de eficiencia, como Barranquilla, Cartagena o Buenaventura.

La Ley 1 de 1991 cambió radicalmente la realidad portuaria colombiana -que había hecho crisis con Colpuertos- al abolir el monopolio estatal con la entrega de los puertos en concesión al sector privado en 1993, la modernización de los mismos y el mejoramiento de su eficiencia.

14 La parte baja del río es navegable, tiene unos ocho pies de calado, allí pueden movilizarse barcazas que tengan económicamente alguna posibilidad para subir de Barranca, inclusive hasta Puerto Berrío en épocas de invierno, pero con muchas limitaciones. 
A pesar del nivel de eficiencia logrado, se tiene que llegar a estándares internacionales. Con el nuevo esquema los operadores portuarios mantienen una libertad de tarifas que no es controlada por la Superintendencia General de Puertos, no obstante no se ha desarrollado el nivel de competencia entre los diferentes operadores que se esperaba en la ley. Y el nivel de inversiones no ha sido suficiente para mejorar la estiba de la carga y los rendimientos de atención a los buques, especialmente en el Puerto de Buenaventura considerado el puerto con las tarifas más costosas del país.

En general, al nivel de infraestructura física, la capacidad de los puertos es limitada, no se dispone de suficiente infraestructura para almacenaje y estiba de la carga manejado por contenedores. En algunos casos la falta de dragado y mantenimiento de las profundidades de calado reducen la posibilidad de que grandes barcos post-panamax arriben a los puertos colombianos. Por último, las zonas francas no cuentan con vías de acceso y servicios que permitan operar como maquilas y transformadoras de bienes a costos competitivos internacionalmente.

Esta falta de eficiencia se explica por el hecho de que los fletes portuarios representan del $4 \%$ al $8 \%$ de los costos totales de transporte, mientras que los fletes terrestres representan cerca del $45 \%$ al $50 \%$ y los fletes marítimos cerca del 45\%. De ahí, que algunos expertos consideran que los esfuerzos que se hagan en materia portuaria afectan en mínima parte la competitividad de los productos colombianos en el mercado internacional.
La concesión del dragado del río Magdalena para mantener la navegabilidad, profundidad y operatividad de los terminales portuarios de Barranquilla, la construcción del Puerto Integrado de Zuñiga para la exportación de los carbones del Cesar, Córdoba y el interior del país, el superpuerto de aguas profundas en Barranquilla, son proyectos que deben adelantarse como parte de un plan de expansión portuaria si hacen más eficientes y competitivas las operaciones y los fletes portuarios.

\section{LA INFRAESTRUCTURA AEROPORTUARIA}

En el sistema aéreo padecemos la crisis que adolece todo el planeta. El sistema interno cualquiera que sea el futuro de las principales aerolíneas colombianas- es un mercado suficientemente fuerte que va a permitir la existencia de empresas aéreas en el futuro. La carga de exportación aérea es limitada, pero muestra un crecimiento dinámico estimulado principalmente por la exportación de flores y otros productos que resisten el flete aéreo. Ahí hay una fortaleza importante.

El tráfico internacional de pasajeros en Colombia aumentó $169 \%$ en la década del 90 como resultado de la apertura económica, un incremento en los viajes de comercio internacional y nuevas alianzas con las naciones del Pacto Andino. No obstante, en materia aeroportuaria el país muestra rezagos por la ausencia de instrumentación aeronáutica de última generación ${ }^{15}$.

Para afrontar ese reto, el gobierno de Colombia ha impulsado varias iniciativas de privatización para desarrollar la infraestructura de transporte aéreo, incluyendo la concesión de la explotación de los aero-
El tráfico internacional depasajeros en Colombia aumentó 169\% en la década del 90 como resultado de la apertura económica, un incremento en los viajes de comercio internacionaly nuevas alianzas con las naciones del Pacto Andino. No obstante, en materia aeroportuaria el país muestra rezagos por la ausencia de instrumentación aeronáutica de última generación.

15 En 1995 la Federal Aviation Administration (FAA) clasificó a los aeropuertos colombianos en Categoría II por razones de seguridad, lo que restringío la posibilidad para las aerolíneas de aumentar el servicio. 
puertos de Barranquilla, Cartagena, Cali y la segunda pista de aterrizaje de Bogotá ${ }^{16}$.

Los cambios en el número de pasajeros domésticos reflejan una relación muy cercana al comportamiento económico de Colombia en términos del PIB. Los pasajeros internacionales, aunque también tienen relación con el PIB colombiano, tienden a ser más influidos por el comercio internacional, el turismo y por factores económicos externos. Por otro lado, a pesar de que se espera un crecimiento continuo de pasajeros en términos absolutos hasta el año 2020, los problemas cíclicos de la economía de Colombia pueden dar como resultado tasas de crecimiento bajas en los próximos años.

Como lo explica la Empresa Boeing en su Market Outlook de 1998, los viajes aéreos internacionales son inducidos parcialmente por el PIB, pero también son inducidos por tarifas reducidas, mejora en los servicios, el PIB de otros países y la globalización económica. Boeing estima que en promedio, por cada $1 \%$ adicional de PIB invertido en el comercio internacional, hay un aumento del 1,5\% en los viajes aéreos.

Para continuar afrontando con éxito el reto de la globalización y el aumento del transporte aéreo se debe mantener la calificación de los aeropuertos colombianos en nivel uno de la FAA y la liberalización tarifaría de este mercado, lo que permitirá a las aerolíneas ofrecer servicios expandidos entre las ciudades de Estados Unidos, Europa, América Latina y Colombia, lo cual refleja el efecto positivo que tiene sobre los pasajeros y la carga internacional.
De igual forma, como proyecto de segundo orden se debe seguir manteniendo y ampliando los aeropuertos regionales dotándolos de una infraestructura adecuada, tanto aeroportuaria como aeronáutica, para asegurar la competitividad regional.

\section{LA INFRAESTRUCTURA URBANA}

En materia de infraestructura urbana, ciudades con una tradición buena en su infraestructura vial como Medellín y Bucaramanga han visto deteriorar su malla vial por la pobreza de los entes territoriales. Bogotá ha sido la excepción, en especial por la construcción de las vías troncales para el transporte masivo y en especial con los nuevos contratos que aseguran un período de mantenimiento de cinco años.

Actualmente existe un programa amplio de renovación de los sistemas urbanos de transporte y de concepción del espacio público, en ciudades como Cali, Pereira, Barranquilla, Cartagena. Así como el programa de expansión de Transmilenio en la ciudad de Bogotá, que incluye algunos de los municipios circundantes. No obstante, en la medida en que el crecimiento de la capital se vea acelerado por las oportunidades que presenta, se hace necesario implementar troncales de mayor movilidad como metros o trenes ligeros, especialmente cuando el volumen de pasajeros en dichas troncales supere las 50.000 personas/hora.

Asimismo, se deben adelantar los proyectos denominados trenes de cercanías ${ }^{17}$, los cuales permitirán el desplazamiento rápido de un número importante de personas que viven en los municipios aleda-

16 Colombia cuenta con 74 aeropuertos grandes de los cuales 21 pueden recibir aeronaves de tamaño B. 17 Se han identificado como viables los trenes de cercanías de 1) Medellín, 2) Cali y 3) la Sabana de Bogotá. 
ños constituidos en ciudades dormitorio, reduciendo la presión urbanizadora sobre las metrópolis, ahorrando en tiempo de desplazamiento y que se traduce en una mejor calidad de vida.

\section{LA INFRAESTRUCTURA ENERGÉTICA}

Es sin duda, el sector que mejor se encuentra para enfrentar el reto de la globalización. La Ley 143 de 1994, la organización del mercado, la disponibilidad de generación, la confiabilidad de los sistemas de transmisión, la regulación del sector, se encuentra al nivel de muchos países desarrollados. Además Colombia cuenta con una situación privilegiada ya que dispone de suficientes recursos en petróleo ${ }^{18}$, gas, carbón e hidroelectricidad que hacen que la oferta actual de energéticos sea variada y no se constituya en un factor que dificulte el desarrollo socio-económico.

El plan de expansión del país se perfecciona con la vinculación de las conexiones internacionales, la responsabilidad en la creación del marco regulatorio del mercado andino de energía y en la meta de hacer del sector privado un actor principal en la expansión del sistema.

En materia de energía existe suficiente oferta, pero se debe avanzar en regulación del mercado de energía transnacional, para exportar los excedentes. La capacidad de generación del sistema alcanzó al 2001 una capacidad efectiva neta de $13.168 \mathrm{MW}$, dis- criminada por recurso energético así: 8.681 MW en plantas hidráulicas, $3.703 \mathrm{MW}$ en plantas de gas, $720 \mathrm{MW}$ en plantas de carbón y $64 \mathrm{MW}$ en plantas de autogeneradores y cogeneradores, que emplean gas, bagazo y otros energéticos.

Existe la teoría del sobredimensionamiento en generación, alimentada en parte por la reducción del consumo interno, pero ante las nuevas demandas que implican las interconexiones con Ecuador y la futura interconexión con Panamá y el resto de Centro América, seguramente ésta aumentará. Este aspecto reviste especial importancia y debe tratarse con sumo cuidado, por cuanto, de producirse un incremento en el consumo que signifique un aumento importante en los precios de la energía, podría traducirse en una merma de la competitividad.

Falta avanzar en electrificación rural, para consolidar la competitividad del agro colombiano. El incremento del abastecimiento de energía en zonas aisladas corresponde a actividades poco rentables. Por tanto, la preocupación del gobierno debe ir más allá de la simple búsqueda de mecanismos, incentivos y/o subsidios, para propiciar un uso productivo de la energía que genere desarrollo regional y local.

Las denominadas zonas no interconectadas $^{19}$, requieren de la implementación de tecnologías más eficientes, limpias y sostenibles que hoy están disponibles. De esta forma, se podrá mantener importantes

18 La suficiencia en materia petrolera está asegurada hasta el año 2007, de no encontrarse nuevos hallazgos de petroleo.

19 Desde el punto de vista del sector eléctrico, Colombia puede dividirse en la región interconectada, que pertenece al Sistema Interconectado Nacional (SIN) y las regiones que por sus características de aislamiento, densidad poblacional no pueden acceder la red de interconexión y se conocen como Zonas No Interconectadas (ZNI).

Según estudios de la UPME, los habitantes de las ZNI son el 4 \% de los colombianos y ocupan el 74 \% del territorio nacional. 
zonas de reserva bioambiental que hacen menos onerosos los costos en la reducción de las emisiones de $\mathrm{CO}_{2}$. Las toneladas de emisiones que se reducen pueden ser "vendidas" en desarrollo de los acuerdos de protección del medio ambiente y su ingreso puede constituirse en una fuente de recursos para este tipo de proyectos.

\section{EL PLAN NACIONAL DE DESARROLLO}

Uno de los objetivos fundamentales del gobierno nacional trazado en el Plan Nacional de Desarrollo 2002-2006, "Hacia un Estado Comunitario" se enfoca a impulsar el crecimiento económico sostenible y la expansión de la infraestructura física del país hacia el mercado interno, desdichadamente sin tener en cuenta la magnitud de la proyección internacional.

La reactivación económica doméstica en el corto plazo necesita esfuerzos focalizados, por lo que el Plan le dará un impulso denodado a la vivienda y a la construcción con el fin de jalonar el crecimiento económico. El Gobierno está comprometido con una reforma regulatoria e institucional en los sectores minero (incluyendo el sector de hidrocarburos), de servicios públicos, de transporte y telecomunicaciones.

En materia de fomento a la vivienda y a la construcción hará ajustes al programa de subsidio familiar de vivienda, otorgará incentivos a la demanda de créditos en UVR y ajustes al sistema de financiamiento de vivienda. Respecto a la exploración y explotación de hidrocarburos y minería, mejorará las condiciones de la actividad petrolera, consolidará la industria del gas, reglamentará los subsidios a combustibles, avanzará en el marco regulatorio de energéticos y fortalecerá el desarrollo del sector minero. En materia de la modernización de la infraestructura estratégica en transporte promoverá la interconexión modal, el mantenimiento y conservación comunitaria de carreteras, dará impulso al transporte urbano y masivo, desarrollará otras modalidades de transporte regional como el fluvial, portuario, aeroportuario y ferroviario. En el tema de servicios públicos domiciliarios, trabajará por optimizar la prestación de los servicios públicos, la reestructuración de empresas de servicios públicos, y en el ajuste de los esquemas de tarifas y subsidios.

Con el objetivo de apoyar el desarrollo en las zonas deprimidas y de conflicto se adelantarán acciones en el área de infraestructura básica, como: a) fortalecimiento del programa Vías para la Paz; b) aumento de la cobertura del servicio de energía eléctrica; y c) desarrollo de soluciones de telefonía comunitaria y de las tecnologías de la información y comunicaciones. Por último, en materia de infraestructura social se apoyará la construcción, rehabilitación y dotación de escuelas, puestos de salud, centros deportivos, recreativos, culturales y de desarrollo comunitario en los municipios más pobres afectados por la violencia.

El Gobierno espera que esta reforma no sólo atraerá mayor inversión extranjera, sino que permitirá, al mismo tiempo, superar los rezagos en infraestructura estratégica que constituyen, actualmente, un obstáculo para el crecimiento económico.

Sin embargo, dado que la estrategia de crecimiento del actual gobierno está sustentada en mayores exportaciones no es muy claro cómo este objetivo se ve reflejado en lo presentado recientemente en el presupuesto general de la Nación. Lo anterior no es nuevo, ya que un componente impor- 
tante de los ajustes fiscales en los países en vía de desarrollo se da por la vía del recorte en la inversión pública incluyendo los gastos de infraestructura.

El tema del presupuesto aún no está totalmente resuelto y existen cuentas sobre las cuales sus ingresos aún no están garantizados. Esto significa en términos prácticos que en caso de tenerse que reducir el presupuesto, se tendría que realizar vía rezago presupuestal. Es decir, posponer planes de inversión en algunos rubros, como por ejemplo, infraestructura, bajando aún más la débil inversión como porcentaje del producto interno bruto.

\section{EL PAPEL DE LA INGENIERÍA NACIONAL}

Colombia cuenta con un portafolio de firmas de excelente calidad y reconocido prestigio que tienen la suficiente capacidad técnica, operativa y financiera para ejecutar el inventario de las obras de infraestructura que necesita el país dentro de niveles internacionales de calidad.

El sector de la ingeniería y la construcción han alcanzado en el ámbito regional un notable posicionamiento en razón de su virtud profesional, empeño empresarial y nivel tecnológico. Esta característica le ha permitido desarrollar grandes proyectos de infraestructura, que se han destacado como obras pioneras de talla mundial. Un catalogo abundante de proyectos ha sido diseñado por ingenieros colombianos, que se clasifican como inigualables, y otras obras han sido construidas por empresas colombianas a la par de las más importantes de la región bajo estándares internacionales de calidad, plazo y precio.

Esta característica pionera de la ingeniería colombiana, se ha fortalecido desde la Constitución de la República, cuando la joven nación colombiana se lanzó temerariamente a la aclimatación en suelo patrio de la compleja tecnología del ferrocarril a vapor, hasta el presente materializada en obras titánicas como el sistema de transporte masivo de Bogotá.

Un número importante de empresas han incursionado con éxito en diseño, ejecución y supervisión de obras en países miembros del Pacto Andino, Centroamérica y el Caribe. Y profesionales independientes han prestado asesorías especializadas en todos los continentes. No obstante el tesón, la tenacidad y la perseverancia de la ingeniería colombiana, se hace indispensable fortalecer este sector con el fin de aprovechar al máximo sus ventajas comparativas para que se constituya en el motor y dinamizador de la economía nacional como lo fue a principios de la década de los noventa.

\section{EL PAPEL DE LA FUNDACIÓN ESCENARIOS}

En este mundo global, en la sociedad global, todo el mundo tiene opciones y las tiene abiertas. Pero se debe competir en precio, calidad y servicio, si no sencillamente no se vende porque hay otros que tienen mejores condiciones. Entonces, lo primero, el país tiene que fijar sus prioridades con toda claridad, no estar sujeto al vaivén de los cambios de gobierno y de los cambios de ministros; creo que tiene que haber un esfuerzo serio y me parece que para la Corporación Escenarios puede ser un tema futuro de reflexión. Si analizamos con cuidado el país, éste nunca se ha sentado a mirar cuales son sus ventajas comparativas, donde debe desarrollarse, qué es lo que debe propiciar como desarrollo competitivo en el ámbito internacional.
Colombia cuentacon un portafolio de firmas de excelente calidady reconocido prestigio que tienen la suficiente capacidad técnica, operativay financiera para ejecutar el inventario de las obras de infraestructura que necesita el país dentro de niveles internacionales de calidad. 
Todo es improvisado, en el gobierno, en el Congreso, inclusive en la industria privada, estamos improvisando y tratando de salir adelante lo mejor que se pueda, pero no hay un plan rector. Planeación Nacional nunca se ha ocupado del futuro, se ha ocupado de resolver los problemas del pasado, y tiene tantos problemas en la agenda del pasado que nunca podrá ocuparse de hacer planeación hacia el futuro.

Creo que este podría ser un tema de la Corporación Escenarios, liderar el proceso de reflexión de qué es Colombia, para dónde va, dónde puede competir en el mundo, cuál es su posición global, dónde debe desarrollarse. Hoy los famosos planes de desarrollo que por ley deben desarrollarse cada cuatro años, no corresponden los unos con los otros, cada uno de ellos improvisa una política para que se satisfaga al gobierno de turno. No hay un hilo conductor que nos lleve al futuro, ni dónde enfocar los esfuerzos; puede ser la minería, pueden ser los servicios, puede ser la agricultura, en fin lo que sea, donde encontremos que tenemos ventaja comparativa y competitiva con respecto al mundo. Pero hoy en día no se sabe, cada uno está haciendo su mejor esfuerzo sin ninguna coordinación con los demás, sin que exista un propósito unificado, sin un libreto único y un plan rector que regule nuestro futuro. Tendría que ser una fundación como Escenarios que está por encima de los intereses particulares, que no pertenece a ningún partido, ni persigue un interés político, que realmente pudiera centrar parte de su esfuerzo y de su actividad en ver qué es el país, para dónde debe ir, ofrecerle al país alternativas de desarrollo que realmente compitan en el mundo y que le den posibilidades de crecimiento.

\section{CONCLUSIONES Y RECOMENDACIONES}

El Banco Mundial ha diagnosticado que el aumento de la infraestructura y el crecimiento económico van a la par. De tal manera que un aumento del $1 \%$ en el desarrollo de la infraestructura va asociado a un crecimiento del $1 \%$ en el PIB.

Así, el nivel de competitividad de un país se mide, entre otros factores, por la calidad y nivel de su infraestructura. En la clasificación del Foro Económico Mundial ${ }^{20}$, Colombia ocupó en el año 2002 el puesto 56 entre 89 países analizados. Está por debajo de economías de la región como Chile (20), Trinidad y Tobago (37), Uruguay (42), Costa Rica (43), México (45), Brasil (46), Panamá (50), República Dominicana (52) y Perú (54) ${ }^{21}$.

Frente al interrogante: ¿Qué tan preparado está el país para la globalización? La respuesta se encuentra sustentada en el análisis de los tipos de infraestructuras físicas, no puede haber competitividad si no hay una infraestructura que la respalde.

20 El Reporte Global de Competitividad (RGC) es la mayor actividad de investigación del Foro Económico Mundial (FEM), concebida como una herramienta internacional para determinar la potencial realización de inversiones y negocios alrededor del mundo. En el RGC se analiza la posición competitiva de un grupo de 59 países compuesto por los 29 países miembros de la OCDE (Organización para la Cooperación y Desarrollo Económico) más otros 30 países seleccionados según el tamaño de sus economías y su importancia relativa en el producto y en el comercio mundiales, desde 1994 Colombia hace parte de estos países.

21 El International Institute for Management Development -IMD- publica cada año el Anuario Mundial de Competitividad (WCY), que es un estudio de la competitividad de las 49 economías industrializadas y emergentes y el cual constituye otro referente para el análisis de Colombia en el contexto internacional. En el tablero de competitividad mundial, Colombia figura en el puesto 16 entre 30 naciones con población mayor a 20 millones. 
Colombia viene enfrentando un retroceso significativo que le reduce progresivamente su capacidad de competir en un mundo cada vez más globalizado. El Plan Nacional de Desarrollo del actual gobierno enfrascado en mitigar los males internos, no tiene claro cómo atender la necesidad creciente de mejorar su infraestructura exportadora para una inserción ordenada de la economía nacional en la economía mundial.

Dentro de este contexto, se puede colegir que es imperativo actuar de forma inmediata para que nuestra economía no sucumba frente a los retos que impone la globalización y el país vea desventuradamente cómo otros países, con menores posibilidades, concretan un mejor futuro al transformar su infraestructura y preparar sus sectores exportables para afrontar con éxito las oportunidades que ofrece el nuevo orden hemisférico y mundial.

Y una de las formas de actuación está en fortalecer el flujo de capitales tanto del sector público como del sector privado para expandir y modernizar nuestra infraestructura. Y para atraer flujos de capital extranjero que complementen el reducido ahorro nacional, es indispensable fortalecer la arquitectura financiera que propicie un clima apropiado para el arribo de dichos capitales, la regulación que asegure unos niveles mínimos de rentabilidad, condiciones atractivas para los futuros concesionarios y con condiciones de estabilidad jurídica que haga atractivo nuestro país cuando se lo compara con los que compiten en la arena global por la inversión extranjera.

\section{SESIÓN DE PREGUNTAS Y RESPUESTAS}

\section{(Interviene el doctor Samper)}

Muchas gracias, Luis Fernando ha brin- dado un panorama muy realista del tema de la infraestructura. Quizás una pregunta: ¿Cuál es la opinión sobre el tema de la financiación? ¿Cómo se podría financiar? ¿Qué dificultades tendría financiar un plan rector de esta naturaleza?, tema que surgió ayer cuando hablamos sobre la financiación para la globalización, las dificultades que hay tanto en el ámbito interno como en el ámbito internacional para la financiación de estas obras.

\section{(Habla el doctor Luis Fernando)}

Presidente, acabamos de pasar por una experiencia en los últimos dos meses que vale la pena ponerla sobre la mesa y revisarla. El Instituto de Desarrollo Urbano de Bogotá diseñó una modalidad de concesión, calificó el riesgo de esa inversión con triple "A", las licitó y esto ha significado que el sector privado financiero colombiano se haya comprometido a invertir dos billones de pesos en la red de Transmilenio de Bogotá en los últimos tres meses. ¿Cómo? Creando confianza, garantizando los recursos necesarios para pagar las obligaciones y colocando las obligaciones con unas tasas de rentabilidad atractivas al mercado. Pero sobre todo el sector financiero es especialmente sensible al tema de la confianza.

Yo lamento muchísimo tener que volver a repetir que si el nuevo gobierno comienza su gestión diciendo que los concesionarios están asociados para defraudar al Estado, que la totalidad de los riesgos deben ser asumidos por los concesionarios, pues así no hay banco o grupo financiero que invierta sus recurso en el sistema concesionado. $\mathrm{Si}$ vamos a cautivar al sector financiero para que invierta en infraestructura, tenemos
Colombia viene enfrentando un retroceso significativo que le reduce progresivamente su capacidad de competir en un mundo cada vez más globalizado. 
que darle condiciones de seguridad, de rentabilidad, de seriedad que lo incentiven a invertir. No podemos estar cambiando las reglas del juego según el capricho de cada gobierno o lo que es peor de cada ministro. Sin embargo creo que se podría lograr que el sector financiero lo hiciera, si creamos el ambiente propicio. Quiero reiterar que las concesiones son en el fondo negocios entre el sector financiero y el gobierno con el papel catalítico de las firmas concesionadas.

El sector financiero internacional sufrió un duro golpe con el fracaso de las concesiones mejicanas que costó mucho dinero a los bancos y a los que habían invertido en el capital de riesgo. Capital que se perdió casi en su totalidad y las concesiones tuvieron que ser reabsorbidas por el Estado mejicano. Este mecanismo tuvo su florecimiento durante el período de Salinas de Gortari, cuando se construyeron cerca de cinco mil kilómetros de autopistas en concesión. Luego en el sexenio siguiente no se hizo prácticamente nada y ahora el gobierno del presidente Vicente Fox ha vuelto a retomar el tema y ha licitado autopistas en concesión con garantía de tráfico y regulaciones que le dan seguridad al sistema financiero de que los recursos que están movilizando los van a recuperar y con una rentabilidad adecuada. Esta nueva política debe mejorar el ambiente internacional de financiación de obra pública.

En el caso colombiano tenemos la dificultad adicional de la violencia y la inseguridad. Sin embargo, estoy seguro de que podemos ir al mercado internacional y al mercado multilateral-BID, Banco Mundial y CAF- a conseguir recursos. Ahora, seguir endeudando al Estado no se puede ya que no tiene una capacidad de endeudamiento ilimitada, estamos llegando a niveles peligrosos de endeudamiento. La única opción es seguir con el sistema que usted desarrolló tan exitosamente en su administración, el de las concesiones y entonces los recursos del sector privado se consiguen, Presidente, con seguridad se consiguen.

\section{(Otra intervención del Presidente Samper)}

Una pregunta sobre el túnel de La Línea: estoy de acuerdo que hacerlo a dos mil seiscientos metros de altitud, no tiene ningún sentido. Iniciar el túnel de ferrocarril a la salida de Calarcá podría ser una alternativa. Hace muchos años atrás se trabajaba en hacer una vía por el sur; por Chaparral, cruzar Palmira por una parte más baja, ¿que tal sería esa opción desde Girardot hasta Buenaventura? Y otra pregunta: como no hay mucho tráfico es muy difícil garantizar niveles de ingreso por peajes. Cómo resolver esa contradicción para que al Estado no le cueste una penalidad enorme cuando dice que en los primeros años van a pasar 10.000 vehículos día, lo que realmente es imposible. Estamos en esa disyuntiva, que para hacer atractivos los proyectos hay que hacer proyecciones optimistas del tráfico y como no pasa inicialmente, pues los concesionarios tienen razón de protestar.

\section{(Vuelve el doctor Luis Fernando)}

Yo creo que en el túnel de la Cordillera Central el problema de la inversión no es solamente en el túnel. Para ir a Chaparral solo hay una parte de la vía puesto que hay que hacer la vía prácticamente nueva, de Girardot desviando a Chaparral y desde allí hasta la boca del túnel, luego a la salida del túnel en el Valle del Cauca, también 
habría que construir una vía nueva y eso costaría una cantidad de dinero posiblemente superior a cualquier alternativa en La Línea.

Lo construido hasta Cajamarca y desde Calarcá hacia el Valle del Cauca tiene especificaciones bastante buenas, y no obstante que hay que hacerle algunas mejorías, la inversión no es una suma desproporcionada. El globo total de la inversión que habría que hacer para tener una vía razonable entre Girardot y Calarcá, por los dos costados, sigue siendo más competitiva todavía por el trazado actual de La Línea. La propuesta que se ha estado revisando, como alternativa a esta propuesta de construir el túnel de los 2.600 metros que no tiene justificación económica, es construir un túnel ferroviario eléctrico más bajo, a unos 1.500 metros, que resultaría de una longitud del orden de los 18 kilómetros. Se tendrían plataformas de cargue y descargue a donde llegarían los vehículos de carga y pasajeros-igual que el túnel de La Mancha o varios túneles europeos- los cuales serían transportados en el tren eléctrico a través de la montaña.

Esta alternativa tiene muchas ventajas: la sección del túnel es menor que la del túnel carretero, el cual tiene problemas de ventilación cuya solución es de por sí muy costosa. Adicionalmente los problemas de seguridad se han constituido en todos lo túneles carreteros importantes de mundo en un tema muy difícil y costoso de resolver ya que cualquier choque es una catástrofe dentro del túnel. En cambio, el túnel ferroviario goza de más seguridad; no tiene problemas de ventilación por ser un tren eléctrico, él mismo sirve como pistón para mover el aire dentro del túnel.
Lo otro es que las concesiones de primera generación fueron estructuradas con unos niveles de tráfico irreales y con esta base se hizo la ingeniería financiera de la concesión y se diseñó el aporte de los concesionarios, la financiación y la contribución del Estado, por medio de los peajes para cancelar la financiación y recuperar el capital de riesgo de los inversionistas con la rentabilidad establecida en el contrato. Con ese modelo se fue al sistema financiero y se consiguieron todos los créditos necesarios para financiar las obras. Luego se hacen las obras y en ese momento se presenta un déficit importante en el tráfico debido a cálculos deficientes y a los problemas de seguridad que afectaron a todas las vías concesionadas. Entonces para que siga siendo viable y equilibrado el modelo financiero y se sostenga la concesión, el Estado tiene que asumir el desequilibrio económico que se presenta mientras se recupera el tráfico.

Las concesiones son a veinte años espero que no sigamos veinte años en esta crisis-, y finalmente el tráfico tendrá que recuperarse y volverá otra vez el sistema a una condición de equilibrio inclusive a producir excedentes. Últimamente el tráfico se ha recuperado, no totalmente, pero ha habido aumentos del orden del 10\% ó $15 \%$ en los últimos meses con todos los programas de seguridad vial. Al hacer el análisis inicial de una concesión por los estructuradores financieros hay que ser totalmente realistas y si el Estado tiene que colocar recursos inicialmente, como lo ha hecho en algunas concesiones, le resulta de todas maneras más económico que aportar los dineros del presupuesto nacional para ejecutar la obra y encargarse del mantenimiento durante veinte años. 
El mantenimiento es otro de los puntos importantes, puesto que todas las concesiones hacen el mantenimiento pesado, o sea poner nuevas capas de rodadura durante todo el tiempo de la concesión. Y hoy en día todas las obras concesionadas están en excelente estado de conservación, en comparación con las vías no concesionadas para las cuales no hay plata para mantenimiento y donde se presenta un deterioro cada vez mayor.

\section{(Interviene un asistente)}

Coincido en lo que expresaba el doctor Jaramillo y es que definitivamente se requiere pensar el país hacia el futuro, desde luego con un sentido estratégico de desarrollo. En ese mismo orden de ideas pensaría qué estamos mirando mucho hacia Estados Unidos y Europa, creo que de todas maneras debemos hacerlo, pero la cuenca del Pacífico es un hervidero de desarrollo y hervidero de comercio y de generación de tránsito y de tráfico internacional. Por otro lado, señor Ministro, me deja preocupado porque tenía esperanzas en el río y en el tren. De todos modos me pregunto esto: ¿cuál sería la importancia y el carácter efectivo y eficiente de la concepción y construcción de puertos secos, como quiera que en la cuenca del río Magdalena está el 70\% y $80 \%$ del país en PIB, en población, en generación de importaciones, en generación de exportaciones; cuál sería el carácter eficiente y efectivo de construir puertos secos en Barrancabermeja, en Puerto Salgar, en la Dorada? Tengo entendido que hay una idea de construir uno en La Tebaida, otro aeropuerto de carga en Cartago.

(Habla el señor Ministro Luis Fernando Jaramillo).
Primero reitero que hay un futuro muy importante para los ferrocarriles donde hay carga, en la movilización del carbón que va a ser uno de los rubros importantes de las exportaciones colombianas hacia al futuro, en la carga a granel y en general en aquellos productos masivos o de características especiales que son los típicos de un ferrocarril. No veo otra opción económicamente mejor que exportar por ferrocarril. El ferrocarril de pasajeros o autoferro, como lo tienen los europeos y en alguna medida los norteamericanos, es muy difícil que con la topografía colombiana pueda competir con un bus de pasajeros climatizado, con baño, bar, televisión, en fin, con todas esas comodidades. La topografía nuestra no permite manejar con facilidad ese tema, de modo que creo que el ferrocarril nuestro está destinado, por lo menos en el futuro próximo, a ser un ferrocarril de carga. Además en el eje del Magdalena podemos tener un ferrocarril donde se muevan dos o tres trenes diarios entre la Dorada y Santa Marta. Eventualmente tenemos que construir las comunicaciones por ferrocarril con los otros dos puertos importantes de la Costa Atlántica, construir la línea hacia Barranquilla y la línea hacia Cartagena, creo que esa es otra de las prioridades del desarrollo de la red férrea.

El transporte multimodal, y aquí tengo unas cifras al respecto, tiene una limitante $y$ es que hay que tener mucho cuidado puesto que cada transferencia que se hace de modo, le significa un costo alto al transporte de la carga. Bajar la carga de Bogotá a Puerto Salgar en contenedores, hacer un transbordo al tren en Puerto Salgar y descargar en Santa Marta es competitivo. Pero si lo bajamos a Puerto Salgar, vamos al tren 
y luego del tren pasamos al río en Barranca o Gamarra, cada movimiento significa costos adicionales y entonces nuevamente, la columna vertebral de cualquier idea de éstas, si es competitiva desde el punto de vista económico, es realizable. Lo más peligroso es jugar con las ilusiones; yo sueño con el ferrocarril, sueño con el río; si no son propuestas económicamente sustentables, no tienen posibilidad práctica de ser ejecutables.

Y el río ha perdido su competitividad por la colmatación, por la cantidad de sedimentos que arrastra, porque bajaron los volúmenes de agua, porque las cuencas están deforestadas, entonces cada vez es menos viable el río. Hoy en día es navegable desde Barranca hasta la Costa, y por eso allí se da el transporte de petróleo y sus derivados en forma competitiva.

Bogotá difícilmente va a tener ferrocarril con la costa por el trazado actual. Hay opciones como el ferrocarril del Carare que podía ser una alternativa hacia el futuro cuyo costo no es tan elevado, es razonable, se estiman alrededor de trescientos millones de dólares.

El aeropuerto de Cartago está funcionando parcialmente como terminal de la carga que genera el eje cafetero, pero Cartago no produce nada, no tiene industrias, no tiene futuro. Ese aeropuerto compite con el aeropuerto de Palmaseca, que en alguna forma parece una talanquera de todo lo que produce el Valle y Cali. Hasta donde me alcanza mi conocimiento, no conozco un aeropuerto exclusivo de carga en el mundo.

\section{(Interpelación del asistente)}

Pero el aeropuerto de Cartago, fue con- cebido con ese propósito, tengo entendido.

\section{(Continúa el doctor Jaramillo)}

Pues claro, esos son los sueños precisamente, fue concebido como un aeropuerto da carga donde no hay carga.

\section{(Interviene el doctor Samper)}

El aeropuerto es viable si se posiciona como el aeropuerto regional del Viejo Caldas. Con la Autopista del Café podrían cerrar el aeropuerto de Pereira, Armenia y Manizales y quedar a 45 minutos o máximo una hora de un aeropuerto que tendría altas especificaciones. Así se justificaría como aeropuerto regional, para mover todo el tráfico de pasajeros, pero Manizales quiere abrir el aeropuerto de Palestina, que no tiene ningún sentido distinto al que puedan viajar de noche como sucedió con el aeropuerto de Rionegro.

\section{(Intervención de otro asistente)}

Tengo simplemente una pregunta, que opina sobre el proyecto de Ley que regula la solución de los conflictos por medio del arbitraje.

\section{(Habla el doctor Jaramillo)}

He estado en estos días discutiendo el tema y el centro de mi inquietud es que no hay justicia oportuna en Colombia. El Contencioso Administrativo no funciona, cualquier pleito que vaya al Contencioso Administrativo se demora entre doce y quince años, esa es en la práctica una denegación total de justicia.

La forma que se ha encontrado en Colombia de resolver este problema los mismo que en muchos países, es la del sistema arbitral. Ahora se está discutiendo un
El río ha perdidosu competitividad porla colmatación, por la cantidad de sedimentos que arrastra, porque bajaron los volúmenes de agua, porque las cuencas están deforestadas, entonces cada vez es menos viable el río. 
proyecto de ley que es un total despropósito. No conozco la última versión que se está discutiendo en la plenaria del Senado, pero lo que se aprobó en la Comisión del Senado, establece que cada arbitramento tiene tres instancias y si el demandado no está satisfecho puede convocar a un nuevo tribunal de arbitramento después de las tres instancias. Nuevamente vamos para seis o siete años lo cual igualmente no es bueno. Además los costos de la Justicia arbitral son bastante altos, para no tener seguridad de que va a fallar definitivamente, que sean fallos vinculantes y sin apelación. El proyecto de ley esta destruyendo el único instrumento que permite que se haga justicia pronta como lo manda la constitución en las disputas entre los particulares o entre estos y el gobierno.

Un problema muy grave, si este proyecto de arbitramento se aprueba como se ha concebido en principio, es que es un golpe definitivo a la inversión extranjera. Nadie va a invertir un solo centavo en Colombia, si no tienen posibilidades de que se haga justicia, de que los conflictos que se generen bien sea entre particulares o en los contratos estatales se resuelvan de una forma expedita.

Cualquiera que sea la opción arbitral o cualquiera que sea la opción de la justicia ordinaria, se va a fallar siempre igual, porque se falla en derecho, y en derecho no puede haber muchas opciones, sino aplicar justicia y si se aplica la justicia tiene que fallarse siempre en una forma determinada. Lo único que debe cambiar es la oportunidad de fallo.

Voy a hacer una última reflexión sobre este tema, me parece que acabar con el sistema arbitral es una de las fuentes de corrupción más importante que puede tener el gobierno, puesto que cuando el fallo va a ser dentro de diez o doce años convierte a los funcionarios públicos en irresponsables, puesto que la justicia nunca los alcanzará . Por el contrario, cuando se va a fallar en seis u ocho meses y el funcionario está viendo a corto plazo el fallo sobre su actuación tiene más cuidado sobre las decisiones que toma ya que existe una responsabilidad fiscal.

\section{(Otra persona interviene)}

El gobierno lo ha presentado como un proyecto para estimular la inversión extranjera, entonces el cuestionamiento es sobre el procedimiento que se establece, ¿acaso no cumple este objetivo?

\section{(Interviene el doctor Jaramillo)}

Claro, el procedimiento hace prácticamente nulo el proceso arbitral, o lo extiende tanto en el tiempo y lo hace tan costoso que deja de ser una opción. Yo le comentaba al Presidente Samper antes de entrar a esta reunión, que anoche había estado reunido con el presidente de una de las empresas constructoras más grandes del mundo. Estaba estimulándolo a participar en los proyectos de Colombia hacia el futuro, como son Porce III en Antioquia, una serie de colectores grandes que se están construyendo en Medellín y Bogotá, el túnel de La Línea, la vía Bogotá Girardot, etc. “A sí, sí, el túnel de La Línea", respondió, "hace diez años he estado pendiente de esta obra con la organización. Le hemos invertido varios millones de dólares en estudios, cada vez que hacemos los estudios y estamos preparados, surge alguna cosa nueva, una 
legislación nueva, un cambio nuevo y todo lo que habíamos hecho se perdió. Te voy a decir una cosa, mientras Colombia siga judicializando la ingeniería, en nuestra empresa no tenemos ningún interés en vincularnos a nuevas obras en Colombia. No tenemos ningún interés en seguir trabajando en Colombia, porque yo soy ingeniero y nuestra empresa es de ingenieros, estamos para trabajar y para hacer las obras y para ganarnos unas utilidades razonables, desde luego, pero no para que todos los conflictos del contrato terminen en un tribunal de arbitramento, muchas gracias, me voy para otra parte donde pueda desarrollar mi actividad básica de ingeniero. No tengo ningún interés en trabajar en nuevas obras en Colombia".

\section{(Intervención de otro asistente)}

Señor Presidente, con relación a uno de los puntos centrales de la intervención del doctor Jaramillo, es el de visualizar el sistema de transporte en Colombia, como elemento estratégico para la competitividad del país, en un contexto globalizado. Me sorprendió descubrir que El Dorado es un aeropuerto que figura entre los principales en América Latina para manejo de carga aérea. Sin embargo allí existen serios problemas que se pueden estar confrontando en cuanto a vías de acceso al aeropuerto, si se proyecta la posible tasa de crecimiento del Dorado; ese es un ejemplo muy concreto de lo que se puede presentar si no se tiene solución estratégica

Un segundo ejemplo es lo que estaba comentando usted, doctor Jaramillo, y es que el aeropuerto de Cali normalmente maneja lo que genera el sur-occidente colombiano, región de influencia de Cali; pero hay un problema muy serio que se está confrontando y es en el sistema logístico de apoyo a la carga aérea, donde hay casos absurdos para los artículos perecederos, como en el caso de las flores tropicales que se están cultivando en el Valle del Cauca. La pérdida era tan grande cuando se estaban exportando a través de Cali, que ahora esos productores del Valle están llevando sus productos a Rionegro y al Dorado, incrementando los costos de transporte, para exportarlas por allí. La deficiencia se incrementó dramáticamente desde que lo está administrando la firma española. Este tema de la exportación de perecederos en Cali, no se ha podido solucionar. Lo que es más gracioso es que, por la legislación colombiana, esas flores están apareciendo como producidas en Antioquia o en Cundinamarca; así se distorsiona la estadística nacional.

\section{(Vuelve a hablar el doctor Jaramillo)}

El problema es complicado y tendrá que irse mejorando en la medida en que aumente la oferta exportable y se creen las facilidades en el aeropuerto. Yo fui exportador de hortalizas hace unos 20 años, teníamos cultivos importantes, de tomate de mesa, de pepino europeo, de pimentón, en fin una variedad grande y nos perjudicó el transporte. Éramos eficientes; una agroindustria organizada, con invernaderos, excelente todo. Bien sea que se quedaban los productos en Bogotá porque no salía el avión, o llegaban a Nueva York en el invierno cuando éramos más competitivos, por cualquier razón no se hacía la nacionalización y se quedaban en patios, llegaba la noche, se congelaba y 
se perdía la totalidad del envío después de que habíamos pagado el transporte aéreo, aduanas, en fin. El transporte es crítico en todo el proceso de artículos perecederos, desde luego, y si no hay volúmenes suficientes no va a haber facilidades para que funcione.

\section{(Interviene el doctor Samper)}

A quien se le ocurre la capital de un país a 2.600 metros de altura y a 1.000 kilómetros de distancia de cualquier costa. Claro que hay una explicación: Llegó Jiménez de Quesada a Bogotá y le dijo a sus amigos, bueno esperemos a que escampe y ahora vemos las consecuencias. Muchas gracias a Luis Fernando, ha sido, sin duda, el bloque más práctico y más puntual de todos los que hemos tenido y ha sido una magnífica oportunidad de revisar el tema de infraestructura.

Damos por terminado nuestro seminario, ha sido uno de los buenos seminarios que hemos tenido, quiero invitarlos a todos a que se hagan presentes en el próximo seminario. Luis Fernando ha dejado una inquietud que vale la recoger, hacer un seminario sobre infraestructura que aborde tema por tema y cuáles son las posibilidades realmente de desarrollo de infraestructura hacia delante. No voy a sacar conclusiones sobre todo a estas alturas simplemente quiero mencionar algo que ya les dije: este es un trascendental esfuerzo que estamos haciendo para que el país piense en otras cosas productivas.

La agenda del país es la violencia, quién está a favor, quién está de un lado, quién está del otro, todo el país está consumido en el tema de la violencia. Si no somos capaces de cambiarle la agenda al país y ponerlo a discutir sobre otros temas, hablarle de la brecha digital, del problema de tecnología, qué vamos a hacer en materia de infraestructura, cuáles son los desafíos de la gobernabilidad o de la educación hacia delante, pues el país va a quedar en angustia esperando a ver quién gana la guerra, si el gobierno o los otros actores del conflicto. Creo que parte de la responsabilidad de quienes tenemos alguna capacidad de orientar al país, es envolverlo en otros temas, como en Chile, Brasil donde se observa que sus periódicos reflejan la realidad internacional, que hay discusiones sobre cuál es el papel que juega la titularización en la financiación de la infraestructura, cómo se está llevando la reunión en Cancún, donde se pueden definir todas las negociaciones comerciales. Entonces creo que ese es un pequeño, discreto y secreto aporte a lo que debe ser un cambio de mentalidad en el país, es cambiarle el chip a la gente, haber si demostramos que hay futuro sin evadir el tema fundamental de la violencia.

Así que quiero consignar una reflexión final. Agradecerles de nuevo a todos ustedes que han sacado parte muy valiosa de su tiempo, y terminar con un gran aplauso por Colombia. 